\title{
Work Environment Factors in Nursing Practice
}

\author{
Aya M. Hegazy ${ }^{1}$, Manal M. Ibrahim ${ }^{2}$, Wafaa A. Shokry ${ }^{3}$ \& Hayam \\ A. El Shrief ${ }^{4}$ \\ ${ }^{1}$ Clinical Instructor of Nursing Administration, ${ }^{2}$ Professor of Nursing Administration, \\ ${ }^{3}$ Assistant Professor of Nursing Administration, ${ }^{4}$ Lecturer of Nursing Administration, \\ Faculty of Nursing, Menoufia University
}

\begin{abstract}
Work environment is the totality of all factors that influence satisfaction, performance, encompasses, intrinsic and extrinsic factors that make a work setting. Conceptual clarity about work environment factors gives empirical direction for future research and a theoretical underpinning for the myriad studies about nurses. Purpose: The present study was conducted for the purpose of clarifying and defining the concept of work environment, its factors, and negative and positive work environment. Methods: in this study, the steps of concept analysis were as follows: Select a concept, determine the aims of the concept analysis, identify various definitions of the concept, determine the concept of Work Environment Factors and identify negative and positive work environment. All studies between the years 2016 and 2021 were reviewed for the purposes of this concept analysis, PubMed, Google search engines, Ovid, and Pro Quest, were scanned and searched using the keywords. Conclusion: The positive work environment could significantly improve organizational outcomes. Identifying factors, which influence the positive environment, may reduce turnover intention and increase work engagement among nurses. These factors include autonomy, environmental control, the relationship between doctors and nurses and organizational support. Nurse Manager need to build a supportive work environment as effective way to increase nurses' psychological bonding and enhancing positive work outcomes that may in turn enhance organizational performance and their work engagement.
\end{abstract}

Keywords: Autonomy, environmental control, organizational support, relationship between doctors and nurses, work environment.

\section{Introduction}

The working environment of nurses is getting global interest and concern because there is a growing consensus that identifying opportunities for improving working conditions in hospitals, it is essential to maintain adequate staffing, high-quality of patients care, nurses' work engagement and minimize their retention (Khan, 2021).

The nurses' work environment is defined as the characteristics of a practice setting that facilitate or constrain professional nursing practice and has been linked to patient outcomes. Nurses' work environment 
plays a key role in the quality and quantity of the care that they can provide as well as in workforce retention. The Study shows that the work environment is related to nurses' intent to leave. When nurses perceived better working conditions, the intent to leave the job decreased and their work engagement increased (McHugh, Rochman, \& Sloane, 2016).

\section{Purpose}

The present study was conducted for the purpose of clarifying and defining the concept of work environment, its factors, and negative and positive work environment.

\section{Methods}

The purpose of concept analysis is to clarify and define the basic elements of a concept. The process allows researchers to distinguish between similarities and differences between concepts. The concept analysis method helps to clarify concepts used in nursing practice that have a broad scope. In this study, the steps of concept analysis were as follows: Select a concept, determine the aims of the analysis, identify various definitions of the concept, determine the concept of environment factors and negative and positive consequences.

\section{Data Collection}

For purposes of this concept analysis, PubMed, Google search engines, Ovid, and ProQuest, were scanned. These databases were searched for the keywords "work environment ", " factors", and "work environment factors in nursing.". All studies between the years 2016 and 2021 were reviewed. Inclusion criteria were: written in English, and described or studied work environment in any setting with any population.

\section{Concept of Work Environment}

Work environment is the totality of all factors that influence satisfaction, performance, encompasses, intrinsic and extrinsic factors that make a work setting. Work environment is acknowledged as a key predictor of work related outcomes, such as higher quality of care and lower turnover intention (Huang et al., 2021).

According the broad view of Edem et al. (2017), the work environment entails employees' safety, job security, good working relationship among employees, recognition for best effort and performance, greatly inspired for performing well and effective involvement in decision-making processes of the organization.

Moreover, the supportive work environment is a key indicator of organizational support for professional employees. Employees yearn for a better work environment, which creates a conducive setting under the support provided by leadership for employee retention (Johnson, Nguyen, Groth, \&White, 2018).

Nursing practice environment is defined as the organizational characteristics of a work setting that facilitate or constrain professional nursing practice (Lake et al., 2019). Nurses' work environment has many aspects: nurses' participation in hospital affairs, nursing foundations for quality of care, nurse manager ability, leadership and support of nurses, staffing and resource adequacy and collegial nurse-physician relations. Improving work environments lead to improve nurses' outcomes and patient outcomes such as job satisfaction, work engagement decreases missed nursing care and patient safety, less burnout, higher quality of care and safer care (Liu et al., 2019).

\section{Work Environment Factors}


The positive work environment could significantly improve organizational outcomes. Identifying factors, which influence the positive environment, may reduce turnover intention and increase work engagement among nurses. These factors include autonomy, environmental control, the relationship between doctors and nurses and organizational support (Rodriguez-Garcia et al., 2021).

Autonomy is a comprehensive issue that requires specific precursors to exist before an individual is considered sovereign. Some antecedents to autonomy are support from senior organizational leadership, personal knowledge and experience, acknowledged competence, leader recognition that the individual has the associated authority to act without barriers and guardrails to provide guidance in decision-making. These factors are paramount for selfgovernance or self- determination in the work place (Lopes, Calapez, \& Lopes, 2017).

Nurses represent the largest percentage of healthcare providers. They play an important role in transforming healthcare. When nurses make autonomous decisions about care, they are questioning the status quo, they are looking to find ways to improve the healthcare system, improve health outcomes, reduce adverse events, and improve patient satisfaction and quality. While providing quality care has always been paramount, quality of care is under particular scrutiny in the current healthcare system. Hospitals and healthcare providers are expected to deliver patient-centered and valuebased care, otherwise healthcare organizations are negatively impacted with financial penalties (Rao, Kumar, \& McHugh, 2017).

The positive consequences of autonomy include increased job satisfaction, increased commitment to the work at hand, position retention, improved work relationships, increased work efficiencies and productivity. The negative impact of autonomy for the organization is that the individual, who feels autonomous, may leave as they have grown in confidence and seek new experiences. Negative consequences of low autonomy levels in nurses such as burnout, depression, job strain, absenteeism and moral distress (Tang, 2021).

Control over the nursing work environment is a democratic process facilitated by a visible, organized and supportive structure. The structure should give nurses input and involvement in decision making concerning clinical policies and problems in practice and personnel issues which affect nurses. The essential control over nursing work environment consists of control over the nursing practice and personnel policies in terms of a structure that enables control, the input and decision making power, recognition of other professionals concerning the control over the nursing practice and the extent to which there is shared decision making and this increase nurses' autonomy which in turn increase their work engagement (Kutney-Lee et al., 2017).

Control-over-practice (environmental control) in the context of this study is defined as the support and opportunity an individual receives so that they may meet their practice expectations in order to provide quality services. Supports include such factors as enough time to discuss client care, sufficient qualified staff and an immediate supervisor who is a good manager and leader. These facets provide an environment where nurses are better able to manage the demands of their practice (Lake et al., 2019).

It was suggested that group practices in primary care foster collaboration with 
other health care providers, which encourages care coordination and leads to a higher quality of primary care. Primarily, nurses were introduced in primary care practices to meet a perceived shortage of primary care physicians (Organization for Economic Co- operation and Development, 2016). Over time, nursing roles and responsibilities expanded. Practice nurses were able to provide holistic care for patients that were not limited to traditional nursing boundaries. Nurses have been found to often provide cost effective patient care and equal high-quality patient care compared to primary care physicians, even with higher patient satisfaction (Pollock, Fernandes, \& Hartling, 2017).

The professions of nursing and medicine together make up a significant portion of healthcare providers. Supporting collaboration between them is essential in the development of appropriate treatment plans that lead to the best patient outcomes. Patients feel the healthcare team is working together to ensure care delivered is carried out appropriately when they visually observe active collaborative measures. A true partnership must be formed to begin a collaborative effort between the nurse and physician. The connection is rooted in trust and best communication practices. The link can be achieved when each profession starts to relate to one another with mutual purpose and respect (Forbes, Larson, Scott, \& Garrison, 2020).

Good working relationship is essential for nurses and doctors to build an effective, safe and conducive environment. These relationships are indeed in teamwork followed by collaboration, communication, respectful and honesty to strengthen the relationship between them. It was produced beneficial towards the patient and their relative in delivering a high quality of care by both of these professions (Tang, Zhou, Chan, \& Liaw, 2018).

In fact, maintaining successful nursedoctor relationships have been shown to be associated with higher satisfaction and better outcomes among hospitalized patients. In contrast, several studies recorded the nurse-doctor relationship negatively influences the nurse's job satisfaction, stress, empowerment, retention and productivity and the outcomes of health care services to patients. The nurses have their own perception of the nurse doctor relationship. With the occurrence of negative thinking or bad behavior, it can affect the nurse-doctor relationship (Nickelsen, 2019).

Globally, all manner of healthcare employees are expected to deliver quality care experiences that keep patient satisfaction scores high. This calls for perceived organizational support to increase the health personnel inner obligation to help the hospitals reach their objectives and their affective commitment to the health care providers. In the converse hospitals can be dangerous places. This compels the management to embrace employee engagement strategies in healthcare as a key strategic tool for righting the ship (Matt, 2020).

However, research shows engagement that is fragmented in the industry, with non-clinical departments exhibiting higher levels while clinical and nursing departments' trend lower. Besides the drivers of employee engagement remain diverse as there are critical differences arising in the way in which each hospital has institutionalized its own unique set of behaviors and actions across its functions, roles and departments (Wang, Liu, Zou, Hao, \& $\mathrm{Wu}, 2017)$.

Organizational support is one of the most important organizational concepts 
that keep employees in the organization, since organizational support is known as a key factor in increasing job satisfaction and the organizational commitment of employees. Employees start having universal opinions about how much their effort are valued and how much the organization cares about their welfare in order to see their organization's readiness to honor employees' contributions and to answer their emotional and social needs (Wen, Huang, \& Hou, 2019).

Perceived organizational support is treated as a guarantee that will help employees fulfill their tasks, do their job efficiently and handle stress. It is also a sign of the relationship between an organization and employees because it tests employees' beliefs about how much their organization rewards employees' efforts and wellbeing (Qi, Liu, Wei, \& Hu, 2019).

\section{Characteristics of Good and Healthy}

\section{Nurses' Work Environment}

Good nurses' work environment is characterized by safe nurse staffing levels, good communication and teamwork with physicians, competent nurse managers and support from hospital management to enable nurses to provide effective and efficient patient care (McHugh et al., 2016). Nurses practicing in good work environment are also highly engaged in the governance and decision-making of their institutions (Garcia-Sierra, Fernandez-Castro, \& MartinezZaragoza, 2016).

Health-care organizations and health policymakers place great emphasis on identifying conditions with the highest impact on achieving better health outcomes. As a major factor in health care, significant efforts have explored the role of the work environment of nurses in enhancing the quality of health-care delivery. Efforts to improve the quality of health-care could result in a healthier workforce, which could benefit the whole community (International Council of Nurses, 2018).

Healthy nursing work environment is defined as a safe, empowering and satisfying workplace, in which all health care staff works conscientiously for optimal health and safety. A healthy work environment provides a fertile ground for effective communication which then contributes to job satisfaction, work engagement and intent to stay. It has been ascertained that nurse satisfaction and patient outcomes are improved in such a healthy work environment (American Nurses Association, 2016).

Healthy work environments (HWE) are characterized by; (a) a high level of trust between management and employees, (b) a culture that supports communication and collaboration, and (c) a climate where employees' feel physical and emotional safety and well- being (Kol, Ilaslan, \& Turkay, 2017).

The American Association of Critical Care Nurses listed six standards that create a healthy work environment: communication, collaboration, effective decision making, appropriate staffing, meaningful recognition and authentic leadership. Excellence in nurse leadership is essential to promoting nurse interpersonal relationships, physical and psychological health and work productivity (Wei, Sewell, Woody, \& Rose, 2018).

\section{Concept and Effect of Positive Work}

\section{Environment Factors}

The American Nurses Association (ANA) has stated that nurses should have a positive work environment, one that is supportive, protective, 
empowering, satisfying and nurturing of optimal health (American Nurses Association, 2017). In recent decades, many countries have restructured, reorganized and re-engineered their health-care systems, and as a result, a positive work environment for healthcare delivery has become an important and powerful factor affecting nurses in clinical practice. Positive work environment has been defined as a work setting in which nurses can achieve organizational goals and to derive personal satisfaction from their work (Lin, Lu, \& Huang, 2016).

The positive environment for nursing practice leads to higher quality patient care may enable nurses to show their professional skills and knowledge and may foster self-actualization among nurses. Finally, a positive work environment may help in increasing employee retention and engagement in a current workplace (Al-Hamdan, Manojlovich, \& Tanima, 2017).

The positive work environment could significantly improve organizational outcomes. Identifying factors, which influence the positive environment, may reduce turnover intention and increase work engagement among nurses. These factors include autonomy, environmental control, the relationship between doctors and nurses and organizational support (Al Sabei et al., 2020).

However, there are negative factors such as increased workloads, an insufficient number of nurses, communication problems within teams, insufficient equipment and a lack of managerial support that result in an unhealthy work environment. Inadequate work environment and negative perceptions are counterproductive to providing highquality and safe health care. Research has linked favorable nursing work environments to better nurse and patient outcomes internationally.
However, many nurses report disengagement and dissatisfaction with their jobs for reasons that can be attributed to the work environment (Olds, Aiken, Cimiotti, \& Lake, 2017). Negative work environment factors, such as irregular shift schedules and having to perform physically and psychologically intense nursing tasks, nurses tend to have an unhealthy lifestyle, which can negatively influence their ability to work. Adverse work characteristics could serve as stressors and barriers to nurses practicing self-care and prevent them from having a healthy lifestyle. For example, extending working hours due to low staffing can result in nurses having insufficient energy to perform self-care behaviors. Besides, negative managerial support can hinder the practice of nurses' health promoting behaviors (Ross, Bevans, Brooks, Gibbons, \& Wallen, 2017). Poor practice environments were found to have negative consequences not only on nurses but also on patients (Swiger et al., 2017).

Rasool, Wang, Zhang, and Samma (2020) established that the moment employees understand that their organization places a high priority on them; they would likely exhibit a greater level of dedication and sense of ownership in the workplace. Diverse elements associated with a working environment such as wages, working hours, employee independence, organizational structure and effective communication between management and its workforce may positively impact on employees' job satisfaction and engagement. When the work environment is not supportive, employees face occupational stress, which leads to negative consequences such as low work engagement, absenteeism and work destruction.

\section{Conclusion}


A conducive work environment plays a vital role in stimulating employees' job satisfaction and engagement, especially in the health-care industry which is plagued with incessant strike actions because of the poor work environment. Such a physical and emotional surrounding defines the working conditions, employee rights, employee voice, and safe conditions of work, cooperative team members and friendly supervisor among others. This concept analysis provides a clearer direction for future research in nurses' work environment.

\section{Implications for Practice}

- Nurse manager need to build a supportive work environment as effective way to increase nurses' psychological bonding and enhancing positive work-related outcomes that may, in turn, enhance organizational performance and their work engagement.

- Encourage individualism and teamwork that aims to respect different ideas and unique skill sets that distinguish nurses from one another.

- In-service training program about nurse practice environment and patient safety for studied nurses need to be conducted.

\section{References}

Khan, N. (2021). Factors that influence nurses' intention to leave adult critical care areas: A mixedmethod sequential explanatory study (Doctoral dissertation, Faculty of Health and Life Sciences, Oxford Brookes University).

McHugh, D., Rochman, F., \& Sloane, M. (2016). Better nurse staffing and nurse work environments associated with increased survival of in-hospital cardiac arrest patients. Med Care, 54(1), 74-80.

Huang, X., Wang, L., Dong, X., Li, B., \& Wan, Q. (2021). Effect of nursing work environment on work related outcomes among psychiatric nurses: A mediating model. Journal of Psychiatric and Mental Nursing, 28(2), 186-196.

Edem, J., Akpan, U., \& Pepple, M. (2017), "Impact of workplace environment on health workers", Occupational Medicine and Health Affairs, 5(2), 1-5.

Johnson, A., Nguyen, H., Groth, M., \& White, L. (2018). Workplace aggression and organizational effectiveness: The mediating role of employee engagement. Australian Journal of Management, 43(4), 614-631.

Lake, Eileen, Sanders, Jordan, Duan, Rui Last,Yong, (2019), "A MetaAnalysis of the associations between the nurse work environment in hospitals and 4 sets of outcomes," Medical care, 57(5), 353-361.

Liu, Jiali, Zheng, Jing, Liu, Ke, You, \& Liming. (2019), "Relationship Between Work Environments, Nurse Outcomes, and Quality of Care in ICUs: Mediating Role of Nursing Care Left Undone," Journal of nursing care quality, 34(3), 250-255.

Rodriguez-Garcia, M., MarquezHernandez, V., GranadosGamez, G., Aguilera-Manrique, G., \& Gutierrez-Puertas, L. (2021). Magnet hospital attributes in nursing work environment and its relationship to nursing students' clinical learning environment and satisfaction. Journal of Advanced Nursing, 77(2), 787-794.

Lopes, H., Calapez, T., \& Lopes, D. (2017). The determinants of work autonomy and employee 
involvement: A multilevel analysis. Economic and Industrial Democracy, 38(3), 448-472.

Rao, D., Kumar, A., \& McHugh, M. (2017). Better nurse autonomy decreases the odds of 30- day mortality and failure to rescue. Journal of Nursing Scholarship, 49(1), 73-79.

Tang, C. Y. (2021). Job Autonomy and Job Performance invention: Work Engagement as a mediator, Age and Perceived Organization Support as a moderator.

Kutney-Lee, A., Germack, H., Hatfield, L., Kelly, S., Maguire, P., Dierkes, A., \& Del Guidice, M. (2017). Nurse Engagement in Shared Governance and Patient and Nurse Outcomes. Journal of Nursing Administration, 46(11), 605-612.

Organization for Economic Cooperation and Development, (OECD). Health at a Glance: Europe. (2016). State of Health in the EU Cycle. 4th edition. OECD publishing; p. 204.

Pollock, M., Fernandes, M., \& Hartling, L. (2017). Evaluation of AMSTAR to assess the methodological quality of systematic reviews in overviews of reviews of healthcare interventions. BMC Med Res Methodol. 17(1), 48.

Forbes H., Larson, K., Scott, S., \& Garrison, G. (2020). Getting work done: a grounded theory study of resident physician value of nursing communication. Journal of interprofessional care, 34(2), 225-232.

Tang, J., Zhou, T., Chan, C., \& Liaw, Y. (2018). Interprofessional collaboration between junior doctors and nurses in the general ward setting: A qualitative exploratory study. Journal of nursing management, 26(1), 1118.

Nickelsen, N. C. M. (2019). The infrastructure of telecare: implications for nursing tasks and the nurse- doctor relationship. Sociology of health \& illness, 41(1), 67-80.

Matt, S. (2020). Employee Engagement in Healthcare: 5 Facts You Need to Know. Retrieved from bonfyreapp.com assesed on 10.02.2020.

Wang, X., Liu, L., Zou, F., Hao, J., \& $\mathrm{Wu}, \mathrm{H}$. (2017). Associations of occupational stressors, perceived organizational support, and psychological capital with work engagement among Chinese female nurses. BioMed research international. 5284628.

Wen, J., Huang, S., \& Hou, P. (2019). Emotional intelligence, emotional labor, perceived organizational support, and job satisfaction: A moderated mediation model. International Journal of Hospitality Management, 81, 120-130.

Qi, L., Liu, B., Wei, X., \& Hu, Y. (2019). Impact of inclusive leadership on employee innovative behavior: Perceived organizational support as a mediator. PloS one, 14(2), e0212091.

Garcia-Sierra, Fernandez-Castro, \& Martinez-Zaragoza. (2016). Work engagement in nursing: an integrative review of the literature, Journal of nursing management, 24(2), 101-111.

International Council of Nurses. (2018). Evidence- based safe nurse staffing. Geneva, Switzerland. Retrieved from https://www.icn.ch/ sites/default/ files/inline-files/ ICNPSEvidencebasedsafenursesta ffing.pdf 
American Nurses Association. (2016). Healthy Work Environment. Available at: http://www.nursingworld.org/M ainMenuCategories/WorkplaceSaf ety/Healthy-Work-Environment, accessed 15 July 2020.

Kol, E., Ilaslan, E., \& Turkay, M. (2017). The effectiveness of strategies similar to the magnet model to create positive work environments on nurse satisfaction. International journal of nursing practice, 23(4), e12557.

Wei, H., Sewell, A., Woody, G., \& Rose, A. (2018). The state of the science of nurse work environments in the United States: A systematic review. International Journal of Nursing Sciences, 5(3), 287-300.

American Nurses Association (ANA). (2017). Healthy Work Environment. Retrived June 15, 2020 , from http://www.nursingworld.org/ Main Menu Categories/ Workplace Safety/Healthy-WorkEnvironment.

Lin, F., Lu, S., \& Huang, Y. (2016). The psychometric properties and the development of the indicators of quality nursing work environments in Taiwan. The Journal of Nursing Research, 24(1), 9-20.

Al-Hamdan, Z., Manojlovich, M., \& Tanima, B. (2017). Jordanian nursing work environments, intent to stay, and job satisfaction. Journal of Nursing Scholarship, 49(1), 103-110.

Al Sabei, D., Labrague, J., Miner Ross, A., Karkada, S., Albashayreh, A., Al Masroori, F., \& Al Hashmi, N. (2020). Nursing work environment, turnover intention, job burnout, and quality of care: the moderating role of job satisfaction. Journal of Nursing Scholarship, 52(1), 95-104.

Olds, M., Aiken, H., Cimiotti, P., \& Lake, T. (2017). Association of nurse work environment and safety climate on patient mortality: A cross-sectional study. International Journal of Nursing Studies, 74, 155-161.

Ross, A., Bevans, M., Brooks, T., Gibbons, S., \& Wallen, R. (2017). Nurses and healthpromoting behaviors: Knowledge may not translate into self- care. AORN Journal, 105(3), 267-275.

Swiger, A., Patrician, A., Miltner, S., Raju, D., Breckenridge- Sproat, S., \& Loan, A. (2017). The Practice Environment Scale of the Nursing Work Index: An updated review and recommendations for use. International Journal of Nursing Studies, 74, 76-84.

Rasool, F., Wang, M., Zhang, Y., \& Samma, M. (2020). Sustainable work performance: The roles of workplace violence and occupational stress. International Journal of Environmental Research and Public Health, 17(3), 912. 